\title{
Beacon-less mobility assisted energy efficient georouting in energy harvesting actuator and sensor networks
}

\author{
Nathalie Mitton ${ }^{1}$, Enrico Natalizio ${ }^{2}$ and Riaan Wolhuter ${ }^{3}$ \\ ${ }^{1}$ Inria, France, ${ }^{2}$ Heudiasyc Lab, UTC, France, ${ }^{3}$ Stellenbosch University, South Africa \\ ${ }^{1}$ nathalie.mitton@inria.fr, ${ }^{2}$ enrico.natalizio@hds.utc.fr, ${ }^{3}$ wolhuter@sun.ac.za *ᄎ
}

\begin{abstract}
In the next years, wireless sensor networks are expected to be more and more widely deployed. In order to increase their performance without increasing nodes' density, a solution is to add some actuators that have the ability to move. However, even actuators rely on batteries that are not expected to be replaced. In this paper, we introduce MEGAN (Mobility assisted Energy efficient Georouting in energy harvesting Actuator and sensor Networks), a beacon-less protocol that uses controlled mobility, and takes account of the energy consumption and the energy harvesting to select next hop. MEGAN aims at prolonging the overall network lifetime rather than reducing the energy consumption over a single path. When node $s$ needs to send a message to the sink $d$, it first computes the "ideal" position of the forwarder node based on available and needed energy, and then broadcasts this data. Every node within the transmission range of $s$ in the forward direction toward $d$ will start a backoff timer. The backoff time is based on its available energy and on its distance from the ideal position. The first node whose backoff timer goes off is the forwarder node. This node informs its neighborhood and then moves toward the ideal position. If, on its route, it finds a good spot for energy harvesting, it will actually stop its movement and forward the original message by using MEGAN, which will run on all the intermediate nodes until the destination is reached. Simulations show that MEGAN reduces energy consumption up to $50 \%$ compared to algorithms where mobility and harvesting capabilities are not exploited.
\end{abstract}

\section{Introduction}

Wireless sensor networks (WSNs) are intended to be deployed in harsh environments. Therefore, it is expected that a large number of cheap sensor devices will be randomly scattered over a region of interest. These devices are powered by batteries and have limited processing and memory capabilities. When batteries deplete, sensors stop covering their area and being part of the underlying communication network. One solution is to add actuators able to move to areas where resources are most needed in order to efficiently route packets between

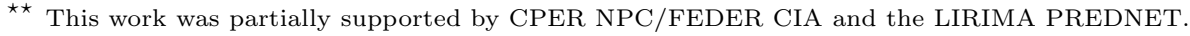


two given nodes. This has been shown [21] that deploying mobile devices in a network can provide the same performance as increasing the nodes' density.

Energy efficient data routing strategies for WSNs have mainly aimed to increase network lifetime $[8,13]$, but none of the most widespread solutions consider energy harvesting. Ambient energy harvesting as a power solution has gained momentum in recent years, especially with significant progress in the functionality of low power embedded electronics. By generating power from environmental energy, the dependency on batteries can be reduced or even eliminated [19].

In this work, we introduce MEGAN (Mobility assisted Energy efficient Georouting in energy harvesting Actuator and sensor Networks) that uses controlled mobility and takes account of the energy consumption (for sending data and moving) and the energy harvesting to select next hop. MEGAN aims at prolonging the overall network lifetime rather than reducing the energy consumption over a single path. Indeed, even if a path selection tries to optimize energy consumption like in $[4,8]$, the same nodes might be selected at each iteration causing a quick depletion of their batteries. In MEGAN, the selection of nodes is based on their current available energy, which could increase or decrease at any time due to energy harvesting. MEGAN has the following characteristics:

- Localized: a node is aware only of its location and that of the destination.

- Memory-less: no information has to be stored at the node or in the message.

- Loop free: nodes choose forwarder among the neighbors which reside in the forwarding direction toward the destination.

- Beacon-less: a node does not need to keep neighborhood tables up to date.

- Energy efficient: MEGAN optimizes the energy consumption along a routing path but also balances the remaining energy over nodes by taking into account the energy consumption for sending and moving, and the harvestable energy. Simulation results show that MEGAN reduces energy consumption up to $50 \%$ compared to some algorithms where mobility and harvesting are not exploited. The rest of this paper is organized as follows. Section 2 reviews literature works. Notations and models used in this paper are detailed in Section 3. Our contribution is detailed in Section 4. Performance evaluation is conducted in Section 5. Finally, Section 6 concludes this work by presenting future works.

\section{Related work}

In this section, we first discuss works about the optimal node placement and movement to allow optimal node positions. Then, we recall algorithms of geographic routing, and finally scan the literature considering energy harvesting.

In this work we are mainly interested in the energy efficiency of the node placement, even though other works focused on other network parameters. The optimal placement of a fixed number of nodes has been mathematically determined in the case of a monodirectional [15] and bidirectional [14] data flow to extend the path lifetime when nodes on the path have different residual energies. All the cited works consider the deployment of static nodes, whereas in our work nodes are mobile and can reach a different and possibly more conve- 
nient location in the field in terms of energy efficiency. The movement of nodes involves an energy expenditure that can negatively affect the overall energy budget, therefore algorithms and heuristics for energy-efficient movements have been proposed [7]. The main difference between our work and the mentioned works is that MEGAN bases the node selection on the energy expenditure for movement and transmission, as well as the harvestable energy.

Unlike MTPR [20] or MMBCR [16] which pursue similar objectives but are on-demand routing protocols (they require a route discovery step), MEGAN is a geographic routing protocol. The basic principle of geographic routing is that each node is aware of its position, the positions of its neighbors and that of the destination. The basic greedy routing has been extended to provide energy efficiency [11], to guarantee delivery [3] and to both provide energy efficiency and guarantee delivery [4]. Nevertheless, all these solutions require a neighbor discovery protocol proved to be very energy-consuming because of required message exchanges [2]. EBGRES [10] is beacon-less like BOSS [18] or BRAVE [1] but differs from these approaches since it takes benefits of controlled mobility. In EBGRES, each node sends out the data packet first. The neighbor selection is processed only among those neighbors that successfully received it. EBGRES uses a 3-way handshake, whereas MEGAN considers a 2-way handshake. In addition MEGAN adapts power transmission to save energy and reduce interferences. These works consider networks composed of static nodes. Current solutions for routing in mobile sensor networks adopt existing routing protocols to find an initial route, and iteratively move each node to the midpoint of its upstream and downstream nodes on the route. However, existing routing protocols may not be efficient. Moving strategy in [5] may cause useless zig-zag movements. In MobileCOP [12], next hop on the path is selected in a cost-over-progress (COP) [11] fashion and then moved to a straight line connecting the source to the destination. Once the first routing has succeeded, nodes on the path are moved and placed equidistantly on the line. Such move may disconnect the network, induces a memory overhead on nodes and a latency. CoMNET [8] was the first solution to propose considering the moving cost into the routing decision and to ensure that node mobility does not disconnect the network. Nevertheless, CoMNET implies a neighborhood discovery, unlike MEGAN, and do not consider the energy that could be harvested. In addition, all these works aim at optimizing the energy consumption along a path and not the consumption of the whole network.

\section{Notations and models}

\subsection{Notation and network model}

We model the network as a graph $G=(V, E)$ where $V$ is the set of nodes, $E$ is the set of edges, and $u v \in E$ if nodes $u$ and $v$ are in transmission range one of each other. Let $N(u)$ be the neighborhood of node $u, N(u)=\{v \mid u v \in E\}$ and $N_{D}(u)$ the set of neighbors of node $u$ in the forwarding direction toward the destination node $D\left(N_{D}(u)=\{v \in N(u) \mid\|\boldsymbol{v}-\boldsymbol{D}\| \leq\|\boldsymbol{u}-\boldsymbol{D}\|\}\right.$ where $u$ 
denotes the identity of node $u$ when $\boldsymbol{u}$ is used for node $u$ position. $\|\boldsymbol{u}-\boldsymbol{v}\|$ is the Euclidean distance between nodes $u$ and $v$.

We assume that every node can adapt its transmission range between 0 and $R_{\max }$ by steps with regards to distance to be reached. Each node $u$ has its own energy level $E_{u}$ such that $0 \leq E_{u} \leq E_{\max }$ where $E_{\max }$ is the maximum energy level that a node can have (same for all nodes). The energy level of node $u, E_{u}$ evolves along time since it can decrease because of sending/receiving message and because of moving. It can also increase based on energy it can harvest from its environment. We will refer to the energy available at node $u$ through the time variable $t, E_{u}(t)$. Note that MEGAN is model-independent but for the sake of evaluation, we use some common energy models computed as follows.

\subsection{Energy models}

Energy model for communication The most common energy model [17] is such that $E_{\text {com }}(d)=d^{\alpha}+c$ if $d \neq 0$ or 0 otherwise, where $d$ is the distance separating two neighboring nodes; $\alpha$ is a real constant $(1<\alpha)$ that represents the path loss; $c$ is a distance-independent term that takes account of signal processing overhead at both the transmitter and the receiver (phase-locked loops, voltagecontrolled oscillators, bias currents, etc.). As in [9] we assume that the energy consumption for this overhead is the same at both sides of the communication.

Energy model for movement To the best of our knowledge, so far, there is no accurate model for defining the energy consumed to move nodes. Therefore, in the following, we use the model adopted in the literature [5,12]: $E_{m_{o v}}(d)=a d$ where $d$ is the covered distance by node $u$ and $a$ a constant to be defined. It only considers the kinetic friction that nodes have to win in order to move, the static friction can be considered simply by adding another constant value.

Energy model for harvesting The amount of energy harvested from the environment can be very different from node to node due to the diversity of harvesters, the locations of the nodes, the deployment policy and the rate of harvesting, etc. The energy model used in this paper is for a solar based harvesting sensor node as defined in [10].

We denote $R_{\text {harv }_{u}}(\boldsymbol{u}, t)$ the rate of energy harvested by node $u$ at time $t$ in position $\boldsymbol{u} . R_{\text {harv }_{u}}(\boldsymbol{u}, t)$ is a deterministic value issued from a previous study of the environment. Node $u$ can operate at time $t$ if its residual energy is greater than the energy needed for communicating or moving at time $t$. If $R_{h a r v_{u}}(\boldsymbol{u}, t)$ is greater that the rate of the energy consumed $R_{\text {cons }_{u}}(t)$, then $R_{\text {harv }}(\boldsymbol{u}, t)-$ $R_{\text {cons }_{u}}(t)$ could be wasted. In these cases we assume that the surplus energy is used to recharge the node battery or can be stored in a capacitor for a later use. In the rest of the paper we will refer to $R_{h a r v_{u}}\left(\overrightarrow{u g_{u}}, t\right)$ as the harvested energy measured by node $u$ on the path between $\boldsymbol{u}$ and $\boldsymbol{g}_{\boldsymbol{u}}$ and averaged over time $t$. We assume that at $t=0$, the battery of the node $u$ is completely charged $E_{u}=E_{\max }$, and that the energy available for node $u$ at any time is given by:

$$
E_{u}(t)=\min \left\{E_{u}(t-1)+\int_{t-1}^{t} R_{\text {harv }_{u}}(\boldsymbol{u}, t) d t-\int_{t-1}^{t} R_{\text {cons }_{u}}(t) d t, E_{\max } .\right.
$$




\section{Contribution}

The idea of MEGAN is to combine the benefits brought by energy harvesting and controlled mobility. MEGAN takes account of several energy components, i.e. the energy spent for sending a message, the energy spent for moving, the energy that can be harvested and the residual energy available at a node to take routing decision. We assume that the transmitter adjusts its transmitting power in order to deliver the minimum required power for a correct reception at the receiver. This allows energy saving. Unlike previous geographical protocols, MEGAN does not assume that its neighborhood is known a priori and thus does not rely in any neighborhood discovery scheme. The idea is the following. We illustrate MEGAN with Fig. 1 and the whole process is summed up by Algo. 1 . We assume that source node $S$ needs to send a packet to destination node $D$, the generic node $u$ chosen by the routing protocol first estimates the optimal position $\boldsymbol{g}_{\boldsymbol{u}}$ of the next forwarder. We will come back on the optimal position computing in the following section.

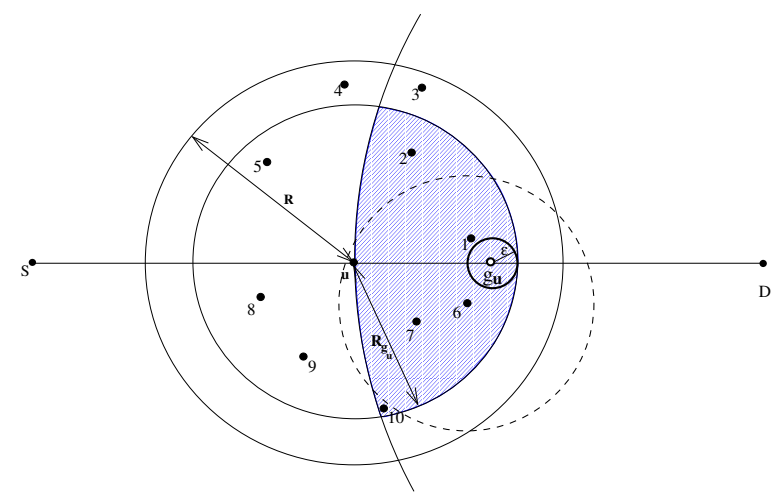

Fig. 1. Example of MEGAN

As a source node, node $S$ runs Algo. 1 only from L. 8. Unlike EBGRES [10], MEGAN takes account of not only the sending energy but also the energy spent for moving, the energy that can be harvested and the residual energy available at node $u$ at current time. Indeed, our goal is not only to have the minimum energy consumption over the routing path but to balance the remaining energy on nodes considering that some of them might harvest more energy than others.

Once $\boldsymbol{g}_{\boldsymbol{u}}$ is computed, node $u$ sends its message $M$ containing $\boldsymbol{g}_{\boldsymbol{u}} \cdot u$ adjusts its range to save energy so that every node located in the circle of radius $R_{g_{u}}=$ $\left\|\boldsymbol{u}-\boldsymbol{g}_{\boldsymbol{u}}\right\|+\epsilon$ around $u$ can be reached. Each generic node $v$ that receives $M$ runs Algo. 1. If $v$ is located in the forwarding direction $\boldsymbol{u D}$ (Dashed area in Fig. 1, nodes $1,2,6,7$ and 10), it computes a backoff time $T_{v}$, otherwise, it discard $M$.

Node $v$ prepares its ACK and waits the backoff time to transmit it. If during the waiting time, $v$ receives an ACK from another node, it discards $M$ (L5-9, 
Algo. 1). Otherwise, node $v$ sends the ACK, to make all other waiting nodes discard $M$. The ACK is used to (i) avoid collisions, since every other candidate will discard the message and (ii) notify $u$ that the greedy routing has succeeded. Node $v$ is the selected forwarder node. $T_{v}$ has been computed in such a way that if $v$ is the first to answer ( $T_{v}$ is the smallest value), it means that $v$ is the closest node to $\boldsymbol{g}_{\boldsymbol{u}}$ (and thus is the one that needs less energy to move to $\boldsymbol{g}_{\boldsymbol{u}}$ ) or if there exists a node $w$ closer to $\boldsymbol{g}_{\boldsymbol{u}}$, its residual energy after the movement would be smaller than the residual energy of $v$ (See Sect. 4.1). Node $u$ waits for an answer from other nodes for a time $T_{\max }$ calculated according to the furthest allowed position $\left(R_{\boldsymbol{g}_{\boldsymbol{u}}}\right)$. If two nodes $v$ and $w$, which are not in the transmitting range of each other, both transmit their ACK to $u$. Since the best forwarder node is the first one that sent the ACK (since it has the lowest backoff time), $u$ advertises all other ones to discard $M$ (L.18-26, Algo. 1).

For instance on Fig. 1, if node 6 is the first one to answer to $u$, it sends an ACK with range $\|\boldsymbol{u}-\mathbf{6}\|$ (dash circle). Nodes 1 and 7 overhear the ACK and thus stop the process. But, this is not the case for nodes 2 and 10 that do not lie in the range of this ACK. Nodes 2 and 10 continue decreasing their back-off time and node 2 sends at its turn an ACK to node $u$. Since this is the second ACK received by $u$ within $T_{\max }, u$ answers node 2 to stop the process (L.24-26, Algo. 1). This ACK is also received by node 10 which also discards $M$.

Node $v$ then moves to $\boldsymbol{g}_{\boldsymbol{u}}$ and based on best energy harvested position adjusts its final position and reiterates the same process (See Section 4.2) Node $v$ reiterates the whole process by first estimating $\boldsymbol{g}_{\boldsymbol{v}}$, the optimal position of the next forwarder, by running Algo. 4. (See Section 4.3) MEGAN is run till delivery to $D$ or till the greedy step fails. In this latter case, a recovery technique is invoked. The design of the recovery step is out of the scope of this paper.

MEGAN is completely distributed and every routing decision is made when needed with regards to the current node topology. It is thus robust to node failure. Dealing with multi-flows is out of the scope of this paper but in such cases, an extension of [6] could be investigated.

\subsection{Back-off computing}

The back-off time should be computed such that the node with more residual energy after the movement to $\boldsymbol{g}_{\boldsymbol{u}}$ be the first node that answers to the routing request of node $u$. Thus, the back-off computation needs to take account of the residual energy of $v$ at the current time $t, E_{v}(t)$, and the energy that the same node will spend for the movement $E_{m o v}$, in order to compute its residual energy after the movement, $E_{v}(t+k)$, where $k$ is the number of time units spent to move to the new location. The energy spent for the movement is computed as detailed in Sec. 3. Once the future residual energy is computed such that $E_{v}(t+k)=E_{v}(t)-E_{\text {mov }_{v}}$, the back-off time, for the node to wait before sending its ACK, is simply the ratio between the maximum energy level $E_{\max }$ and this value. In fact, since $0<E_{v}(t+k) \leq E_{\max }$ then the back-off time will be $1 \leq$ $\frac{E_{\max }}{E_{v}(t+k)}<\infty[\mathrm{ms}]$. Nodes that calculate a back-off larger than a given threshold can just discard the routing request and avoid replying to $u$. Let us assume on 


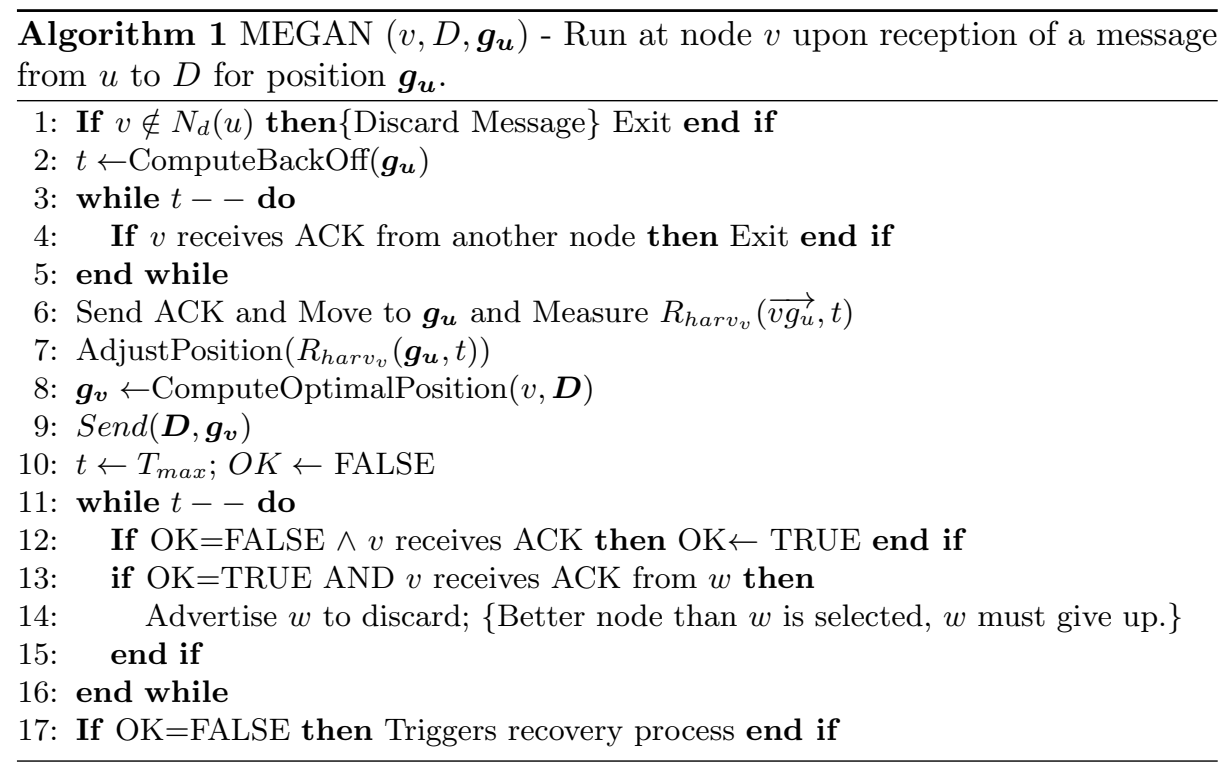

Fig. 1 that node $u$ sends its routing request in a radius $R_{\boldsymbol{g}_{\boldsymbol{u}}}$. All nodes $v$, such that $\|\boldsymbol{u}-\boldsymbol{v}\|$, (nodes $1,2,5,6,7,8,9,10$ ) receive it. Only nodes in the forwarding direction of $D$, (nodes $1,2,6,7,10)$ triggers a back-off time. Let the following values for the energies at time $t: E_{1}(t)=4, E_{6}(t)=7, E_{\text {com }}\left(\left\|\mathbf{1}-\boldsymbol{g}_{\boldsymbol{u}}\right\|\right)=2$ and $E_{\text {com }}\left(\left\|\boldsymbol{6}-\boldsymbol{g}_{\boldsymbol{u}}\right\|\right)=4$. After $k$ time units needed for the physical movement, we would have $E_{1}(t+k)=4-2=2$ and $E_{6}(t+k)=7-4=3$. So, even if 6 is further to $\boldsymbol{g}_{\boldsymbol{u}}$ than 1, it will be chosen. This favors the balance of energy consumption over nodes.

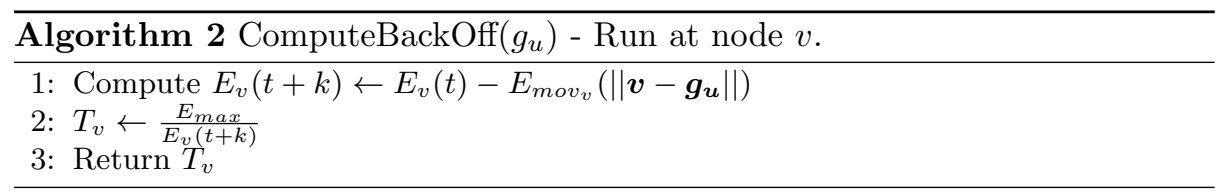

\subsection{Position adjustment}

When node $v$ (that is in position $\boldsymbol{v}$ before the movement) moves to the estimated optimal position $\boldsymbol{g}_{\boldsymbol{u}}$, it can instantaneously measure the energy harvesting rate in its new position. During its movement, it has retrieved information about the $R_{\text {harv }_{v}}\left(\overrightarrow{v_{u}}, t\right)$ in all the intermediate positions between its original position $\boldsymbol{v}$ and position $\boldsymbol{g}_{\boldsymbol{u}}$. Through all this information, when $v$ reaches $\boldsymbol{g}_{\boldsymbol{u}}$, it can decide to move of $\epsilon$ in one of the following four directions (as shown on Fig. 2): 
1. $\overrightarrow{g_{u} u}$, i.e. toward $u$;

2. $\overrightarrow{u g_{u}}$, i.e. against $u$;

3. $\overrightarrow{v g_{u}}$, i.e. it keeps going in the current travel direction;

4. $\overrightarrow{g_{u} v}$, i.e. it backtracks.

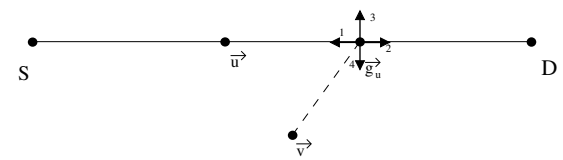

Fig. 2. Position adjustment

Intuitively, in ideal conditions of node density and residual energy, $\overrightarrow{u g_{u}}$ would be directed as the vector $\overrightarrow{S D}$ and $\overrightarrow{v g}$ would be perpendicular to $\overrightarrow{S D}$. Therefore, the four mentioned directions would represent the extreme points of two perpendicular diameters of the circle having $\boldsymbol{g}_{\boldsymbol{u}}$ as the center and $\epsilon$ as the radius, as shown in Fig. 1. This means that the position adjustment algorithm, in ideal conditions, can take $360^{\circ}$ around $\boldsymbol{g}_{\boldsymbol{u}}$ into account. Direction 1 is chosen when the energy harvested in $\boldsymbol{u}$ is significantly larger than that harvested in $\boldsymbol{g}_{\boldsymbol{u}}$ and the gradient of the $R_{h a r v_{v}}$ along $\overrightarrow{v g_{u}}$ is not significantly different from 0 . Direction 2 is chosen when the energy harvested in $\boldsymbol{u}$ is significantly smaller than that harvested in $\boldsymbol{g}_{\boldsymbol{u}}$ and the gradient of the $R_{h a r v_{v}}$ along $\overrightarrow{v g_{u}}$ is not significantly different from 0 . Direction 3 is chosen when the gradient of the $R_{h a r v_{v}}$ along $\overrightarrow{v g_{u}}$ is significantly positive and the energy harvested in $\boldsymbol{u}$ is not significantly different from that harvested in $\boldsymbol{g}_{\boldsymbol{u}}$. Direction 4 is chosen when the gradient of the $R_{\text {harv }_{v}}$ along $\overrightarrow{v g_{u}}$ is significantly negative and the energy harvested in $\boldsymbol{u}$ is not significantly different from that harvested in $\boldsymbol{g}_{\boldsymbol{u}}$. For the fourth case, a further optimization would make node $v$ stop its movement in the direction $\overrightarrow{v g_{u}}$, instead of making it reach $\boldsymbol{g}_{\boldsymbol{u}}$ and then backtracks.

When the gradient of the $R_{h a r v_{v}}$ along $\overrightarrow{v g_{u}}$ is zero and the energy harvested in $\boldsymbol{u}$ is not significantly different from that harvested in $\boldsymbol{g}_{\boldsymbol{u}}$, then $v$ does not move from $\boldsymbol{g}_{\boldsymbol{u}}$. On the contrary if both the gradient and the mentioned difference are significantly high then the node will move simultaneously along both the axes. In order to quantify the differences between the energy harvested along the direction $\overrightarrow{S D}$ and that perpendicular to $\overrightarrow{S D}$ we introduce two threshold values, $H_{p a r}$ and $H_{p e r}$, respectively. For sake of simplicity in Algo. 3 we omitted the time variable.

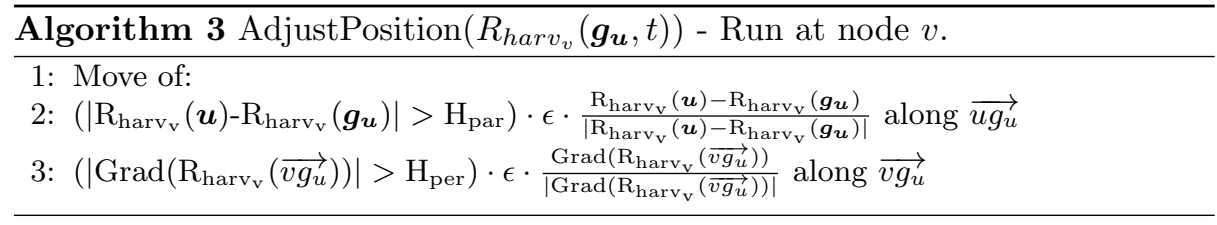




\subsection{Computing of optimal position for forwarder node}

In order to estimate the optimal position for next node in the path towards the destination node $D$, we assume that node $v$ is aware of $E_{\max }$, its energy at current time $t, E_{v}(t)$, its energy consuming and harvesting rates, $R_{\text {cons }_{v}}$ and $R_{\text {harv }_{v}}$, respectively. By multiplying the energy consuming and harvesting rates for a certain number of $\Delta t$ time units, $k$, node $v$ can estimate its future residual energy, $E_{v}(t+k \Delta t)$, available after $k$ time units. If this value does not decrease in respect of the current residual energy $\left.\left(E_{v}(t+k \Delta t) \geq E_{v}(t)\right)\right)$, then the ideal position, computed from the optimal transmission radius, is given by:

$$
\boldsymbol{h}_{\boldsymbol{v}}=\boldsymbol{v}+d^{*} \cdot \frac{\boldsymbol{D}-\boldsymbol{S}}{\|\boldsymbol{D}-\boldsymbol{S}\|}
$$

In this case, $h_{v}$ is also the optimal position $\boldsymbol{g}_{\boldsymbol{v}}$ for next relay $\left(\boldsymbol{g}_{\boldsymbol{v}}=h_{v}\right)$. Otherwise (if $\left.E_{v}(t+k \Delta t) \leq E_{v}(t)\right)$ ) an intermediate position will be considered as the estimated optimal position. Notice that several approaches could be considered in order to estimate next forwarder optimal position, depending on the criteria of optimality. The definition of the criteria of optimality is out of the scope of this work. Hence, we introduce two simple approaches depicted in Algo. 4 based on energy computation: a conservative approach that keeps the forwarder node close to $\boldsymbol{v}$, and an optimistic that pushes it close to position $v^{e c t} g_{u}$ when $\left.E_{v}(t+k) \geq E_{v}(t)\right)$. We thus have:

$$
\boldsymbol{g}_{\boldsymbol{v}}= \begin{cases}\left(\frac{E_{v}(t)-E_{v}(t+k \Delta t)}{E_{m a x}}\right) \cdot h_{v} & \text { if conservative approach } \\ \left(1-\frac{E_{v}(t)-E_{v}(t+k \Delta t)}{E_{\max }}\right) \cdot h_{v} & \text { if optimistic approach }\end{cases}
$$

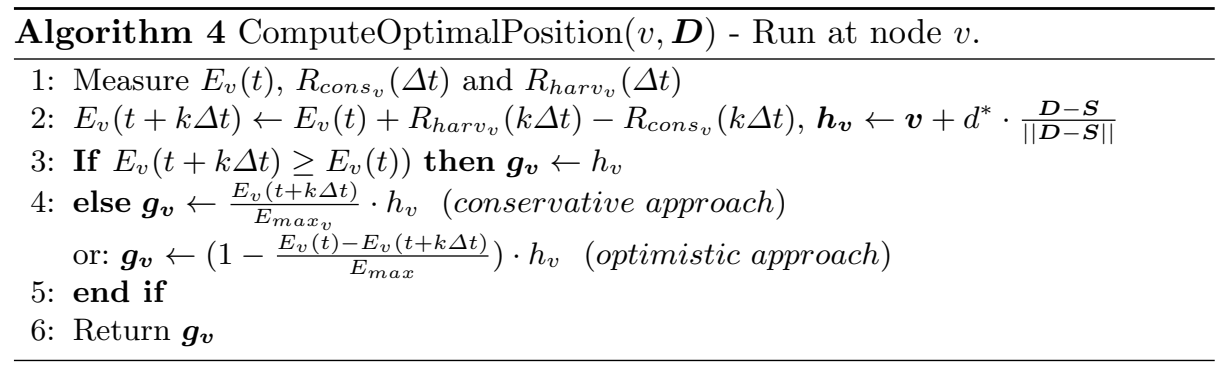

\section{Simulation results}

To evaluate the performance of MEGAN, we run the simulation in OMNET++ with a CSMA/CA MAC layer. We assume a free space propagation model and packet collisions to carry out the tests, and that when a node dies it cannot be resuscitated. Sensor nodes are uniformly spread throughout a square area of $1000 \times 1000 \mathrm{~m}^{2}$ and they can adapt their transmission range between 0 and $100 \mathrm{~m}$. Only connected networks are considered in the results. The DATA message 
payload is set to 128 bytes, the size of the ACK message is 25 bytes. We use the cost models described in Section 3 with the propagation loss exponent $\alpha$ set to 2 and $c=50 \mathrm{~nJ} / \mathrm{bit}$. We use two values of $a$ for the cost model related to movement : 0.1 and 10, to make the energy to send prevail over the energy to move and vice versa, respectively and we simulate two scenarios accordingly. Each sensor node is initialized with 2J of battery energy.

The transmission range depends on the energy available at the node and the transmission delay. We are mainly interested in evaluating the total energy consumption for sensor-to-sink packet delivery independently from the MAC layer used. We conducted 50 replications of each simulation scenario to obtain statistically significant output. In each replication, each of the 20 nodes, which are randomly chosen as sources, generates 40 data packets to be transmitted to a randomly chosen destination. The simulation terminates when all the data packets generated in the network are delivered. We study the evolution of the cumulative energy spent to send (and receive) all the data and control packets in the network, when both the transmission range and the nodes' density vary.

We compare MEGAN with EBGRES and COMNET, because those are the two conceptually closest algorithms to MEGAN, which is both a beacon-less energy harvesting routing scheme, as EBGRES, and a mobility based routing scheme, as COMNET.

Energy consumption versus transmission range Fig. 3 shows the total energy expenditure of the network for both the scenarios (sending cost higher than moving cost, and vice versa), when the transmission range varies. In both cases, MEGAN outperforms the other protocols. We can see that, for all the algorithms, the energy expenditure decreases exponentially when the transmission range increases, because a smaller number of intermediate nodes is needed to cover the source-destination path.

Both MEGAN and COMNET are mobility based algorithms, thus they perform best in cases that favor mobility (Fig. 3 (a)). MEGAN consumes less energy than COMNET because it is a beacon-less approach and has less message handshakes needed to set up the transmission path. Furthermore, in comparison with MEGAN, COMNET uses a flooding approach for neighbor discovery and is not reactive. When sending data is more convenient than moving nodes (Fig. 3 (b)), COMNET consumes too much energy to move to the optimal position, whereas MEGAN moves in a more intelligent way and saves enough energy to perform better than EBGRES to send the same amount of data.

Energy consumption versus nodes' density Fig. 4 shows the total energy expenditure of the network for both the scenarios, when the transmission range is set to $100 \mathrm{~m}$, and the nodes' density varies. We can see that, for all the algorithms, the energy expenditure decreases linearly when the nodes' density increases, because, for all the simulated algorithms, the selection procedure has more chances to find a relay node close to the optimal position. We can see from Fig. 4 (a) that when sending data is more costly than moving nodes, EBGRES and COMNET perform almost identically. Whereas, from Fig. 4 (b), we can see that when moving nodes is more costly than sending data EBGRES performs 


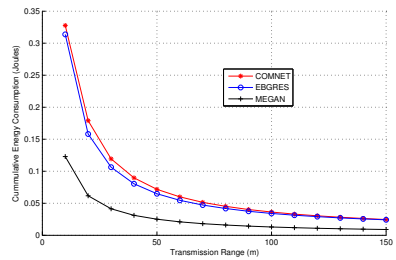

(a) Moving $<$ Sending

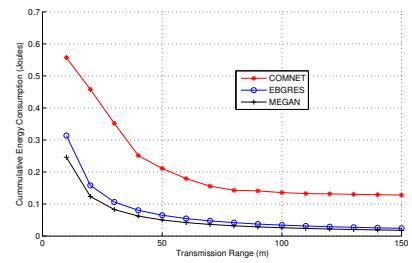

(b) Moving $>$ Sending

Fig. 3. Cumulative energy consumption versus transmission range

better than COMNET. However, in both the scenarios, MEGAN spends less energy than the other protocols, due to the mentioned reasons of using mobility in a smarter way than COMNET and of requiring less control messages than EBGRES as well as of finding better positions than EBGRES to forward data. An interesting remark is that when moving nodes is "expensive" the energy saved by finding a better position in MEGAN for each intermediate node is compensated by the additional energy needed to move to that position. In fact, EBGRES and MEGAN almost overlap in this scenario.

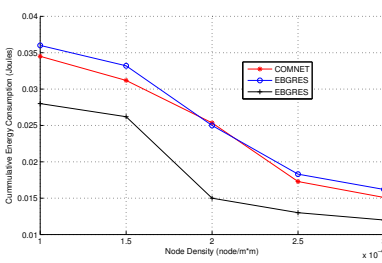

(a) Moving < Sending

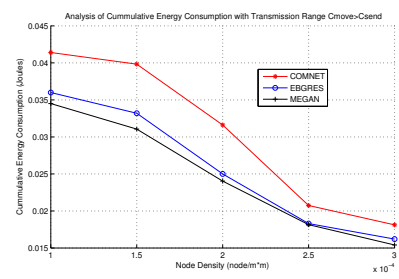

(b) Moving > Sending

Fig. 4. Cumulative energy consumption versus node density

\section{Conclusion and future works}

In this paper, we introduced MEGAN, the very first beacon-less geographic routing that takes advantage of controlled mobility and energy harvested from the environment to dynamically adapt the energy consumption. Simulation results show that MEGAN outperforms literature protocols by reducing up to $50 \%$ the energy consumption under some parameters.

As a next step, we intend to investigate recovery mechanisms that also takes account of these hardware characteristics to improve the routing performances.

\section{References}

1. I. Stojmenovic A. Khan, C. Constantinou. Realistic physical layer modelling for georouting protocols in wireless ad-hoc and sensor networks. In ICUMT, 2009. 
2. I. Amadou, G. Chelius, and F. Valois. Energy-Efficient Beacon-less Protocol for WSN. In PIMRC, 2011.

3. P. Bose, P. Morin, I. Stojmenovic, and J. Urrutia. Routing with guaranteed delivery in ad-hoc wireless networks. ACM/Kluwer Wireless Networks, 7(6):609-616, 2001.

4. E. H. Elhafsi, N. Mitton, and D. Simplot-Ryl. End-to-End Energy Efficient Geographic Path Discovery With Guaranteed Delivery in Ad hoc and Sensor Networks. In IEEE PIMRC, 2008.

5. D.K. Goldenberg, J. Lin, and A.S. Morse. Towards mobility as a network control primitive. In Mobihoc, pages 163-174, 2004.

6. N. Gouvy, E. Hamouda, D. Zorbas, and N. Mitton. Energy Efficient Multi-Flow Routing in Mobile Sensor Networks. In IEEE WCNC, Shanghai, China, 2013.

7. F. Guerriero, A. Violi, E. Natalizio, V. Loscri, and C. Costanzo. Modelling and solving optimal placement problems in wireless sensor networks. Applied Mathematical Modelling, 35(1), 2011.

8. E. Hamouda, N. Mitton, and D. Simplot-Ryl. Energy efficient mobile routing in actuator and sensor networks with connectivity preservation. In AdHocNow, 2011.

9. W.B. Heinzelman, A.P. Chandrakasan, and H. Balakrishnan. An applicationspecific protocol architecture for wireless microsensor networks. IEEE Trans. on Wireless Com., 1(4), 2002.

10. O. Jumira, R. Wolhuter, and S. Zeadally. Energy-efficient beacon-less geographic routing in energy harvested wireless sensor networks. In Concurrency and Computation: Practice and Experience, 2012.

11. J. Kuruvila, A. Nayak, and I. Stojmenovic. Progress and location based localized power aware routing for ad hoc sensor wireless networks. IJDSN, 2:147-159, 2006.

12. H. Liu, A. Nayak, and I. Stojmenovic. Localized mobility control routing in robotic sensor wireless networks. MSN, 4864:19-31, 2007.

13. S. Momma, T. Mikoshi, and T. Takenaka. Power aware routing and clustering scheme for wireless sensor networks. In APSITT, 2010.

14. E. Natalizio, V. Loscri, F. Guerriero, and A. Violi. Energy spaced placement for bidirectional data flows in wireless sensor network. IEEE Com. Letters, 13(1), 2009.

15. E. Natalizio, V. Loscri, and E. Viterbo. Optimal placement of wireless nodes for maximizing network lifetime. IEEE Com. Letters, 12(5), 2008.

16. H. Nishat, D. Srinivasa Rao, and C. Balaswamy. Energy efficient routing protocols for mobile adhoc networks. Int. Journal on Computer Applications, 26(2), 2011.

17. V. Rodoplu and T. Meng. Minimizing energy mobile wireless networks. IEEE JSAC, 17(8):1333-1347, 1999.

18. J. A. Sánchez, R. Marín-Pérez, and P. M. Ruiz. Boss: Beacon-less on demand strategy for geographic routing in wireless sensor networks. In MASS, 2007.

19. C. M. Vigorito, D. Ganesan, and A. G. Barto. Adaptive control of duty cycling in energy harvesting wireless sensor networks. In SECON, 2007.

20. D. Vir, S.K. Agarwal, S.A. Imam, and L. Mohan. Performance analysis of MPTR routing protocol in power deficient node. Int. Journal on AdHoc Networking Systems (IJANS), 2(4), 2012.

21. W. Wang, V. Srinivasan, and K.-C. Chua. Extending the lifetime of wireless sensor networks through mobile relays. IEEE/ACM Trans. Netw., 16(5):1108-1120, 2008. 